\title{
An Improved Method for Microwave Power Calibration, With Application to the Evaluation of Connectors*
}

\author{
Glenn F. Engen* * \\ Institute for Basic Standards, National Bureau of Standards, Boulder, Colorado 80302
}

(April 23, 1971)

\begin{abstract}
In the procedures for microwave power calibration, which are well documented, the subject of mismatch errors (or corrections) plays a major role. In particular, the evaluation of mismatch corrections requires the measurement of complex reflection coefficients; and the accuracy of this measurement is limited, in part, by connector imperfections.

The application of recently developed "power equation" methods to this problem provides both a simplified determination of the mismatch correction $\left(\mathrm{M}_{\mathrm{gm}}\right)$ and improved accuracy. In particular, the intermediate step of measuring the reflection coefficients is eliminated, and the precision connector requirement is greatly relaxed. If this new method were adopted at each level of the usual calibration hierarchy, the accuracy of disseminating measurements referenced to the primary standards at NBS would be greatly improved.

The accuracy potential was demonstrated in a series of experiments involving the Type $\mathrm{N}$, GPC-7, and waveguide flange connectors. The outcome of this experimental evaluation, in which the "ordinary" Type $\mathrm{N}$ performed on a par with GPC-7, raises some rather serious questions relative to current trends in connector development.
\end{abstract}

Key words: Calibration; connectors; mismatch; power equation; power measurement.

\section{Introduction}

The use of a calibration heirarchy, as means of disseminating measurements referenced to the primary standards at NBS, is a long established practice. Unfortunately, it is a general and apparently unavoidable feature of calibration heirarchies that the measurement accuracy is degraded at every step between the primary reference laboratory (NBS) and the field working level.

In the case of microwave power, the calibrated items are usually bolometer mounts or directional coupler-bolometer mount combinations. Here the practical problems, in comparing one mount against another for example, are substantial, and the relative loss in accuracy at each step is much larger than occurs in other parameters such as mass or d-c voltage. Indeed, although reliable data are not available, the probable loss in accuracy appears to be such that serious questions have been raised about the usefulness of the calibration heirarchy (for this parameter) other than that of satisfying the contractual requirement for measurement "traceability."

Much of this difficulty centers around the problem of "mismatch" corrections. Until recently, there has been no simple and direct method of measuring and applying this correction. The approach has sometimes been a hybrid one of adjusting for a generator match, then measuring the other parameters. More often, particularly at the lower levels, this correction is ignored and thus becomes an error whose magnitude depends upon the attendant impedance parameters, and whose total value may be sub- tantial if permitted to accumulate throughout the hierarchy.

It is the purpose of this paper to review this problem from the viewpoint of the recently developed "power equation" technology $[1]^{1}$ and present a simplified, yet more accurate, method of measuring the mismatch factor.

\section{General Description}

The measurement problem is conveniently visualized with the help of figure 1. The measurement cycle usually begins with a "standard" power meter which may be either in the form of a calorimeter $[2]$, or of the bolometric type, where the bolometer mount properties have been evaluated by microcalorimetric [3] or impedance [4] methods. The standard power meter is used to calibrate a signal generator, this, in turn, is used to calibrate another power meter. This item is then sent to another calibration laboratory where it becomes the reference standard and the process is repeated.

With regard to the signal generator calibration, it may be noted that microwave generators do not ordinarily possess sufficient stability to warrant their calibration as such. The signal generator calibration, which is obtained in the calibration transfer from one power meter to another, may thus be of interest only during the measurement procedure and not even explicitly extracted from the

\footnotetext{
*Presented at the 1970 Fall Meeting of URSI, September 15-17, Ohio State University, Columbus, Ohio, 43210.

**Electromagnetics Division, Senior Research Scientist, National Bureau of Stand. ards, Boulder, Colo. 89302

${ }^{1}$ Figures in brackets indicate the literature references at the end of this paper.
} 


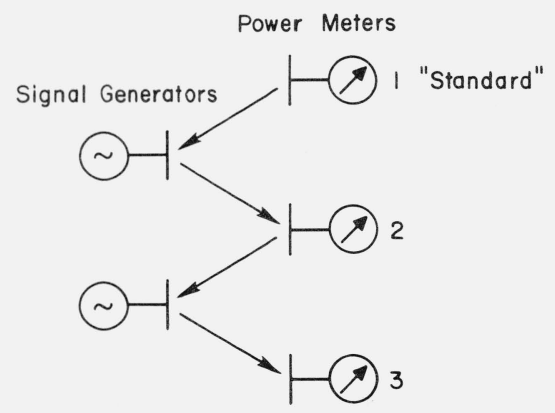

FIGURE 1. Illustrating the power calibration hierarchy.

measurement data. There is no loss in generality, however, in describing the procedure in this framework.

Alternatively, the signal source may be provided with an output monitor-in the form of a directional coupler and side-arm power meter, for example. Here the "generator calibration" is in reality a calibration of this output monitor, and is usually of future interest. This, in fact, is the recommended mode of operation and will be explained in greater detail in what follows.

Returning to figure 1, it is tempting, and in many cases correct, to interpret the column of power meters as the "working" or "interlaboratory" standards for the several echelons in the calibration hierarchy. It is also noted, however, that the number of calibration steps is reduced by approximately one-half when the calibrated signal generator is adopted as the working standard at the alternate levels. This should also reduce the error accumulation, but at present, there is no universally accepted practice in this matter.

Each step of the "power equation" calibration procedure is described by the equation,

$$
\mathrm{P}_{g m}=\mathrm{P}_{g} \mathrm{M}_{g m},
$$

where $\mathrm{P}_{g m}$ is the (net) power absorbed by the meter, $\mathrm{P}_{g}$ is the available power from the signal generator, and $\mathbf{M}_{g m}$ is a "mismatch" factor which expresses to what extent the conditions for maximum power transfer between generator and termination (power meter) are satisfied. It should be noted that $\mathrm{P}_{g}$ is a property of the generator only, while $\mathbf{M}_{g m}$ (and thus $\mathbf{P}_{g m}$ ) is a property of the signal generator and power meter combination.

In the first step, the "first" generator and standard power meter are mated. Subject to certain efficiency corrections, the standard power meter indicates $\mathrm{P}_{g m}$, and if $\mathbf{M}_{g m}$ is also known, $\mathbf{P}_{g}$ may be obtained from (1).

In the second step, the first generator is connected to the second power meter. Assuming the $\mathbf{M}_{g m}$ for this combination of components is measured, $\mathrm{P}_{g m}$ may be determined from (1) since $\mathrm{P}_{g}$ is a property of the generator only. The calibration of this second meter is now obtained by comparing its reading with the value of $\mathrm{P}_{g m}$ thus obtained. This process is repeated at each calibration laboratory. It will be immediately recognized that the determination of $\mathrm{M}_{g m}$ plays a major role in this procedure.

\section{Measurement of $\mathbf{M}_{\mathrm{gm}}$}

As already noted, the recommended "signal generator" includes an output monitor which is usually a directional coupler with a bolometric detector or power meter on the sidearm as shown in figure 2 . It is convenient (but not essential) to postulate feedback such that this sidearm power is constant. It has been shown [5] that the impedance of the "equivalent generator" which obtains at the output port (2) depends only on the coupler parameters and is independent of the actual signal source. The available power, at the output port, also depends only on the coupler parameters and is linearly related to the sidearm power level. For these reasons, it is convenient to interpret the measurement of the pertinent coupler parameters as a signal generator calibration.

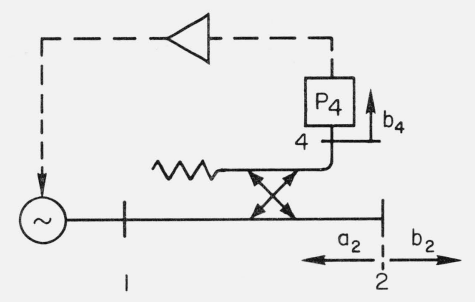

Figure 2. Signal generator with output "Monitor"

The sidearm detector and power meter to be calibrated are often of the bolometric type. Although the attendant problems are by no means trivial, they are well understood and the associated instrumentation is highly developed [6]. The major existing problems are associated with the measurement of $\mathrm{M}_{g m}$. Here the power meter mismatch, imperfect coupler directivity, connector imperfectons, etc. enter the picture.

A measurement of $\mathrm{M}_{g m}$ may be effected by the addition of a second coupler, power detector $\left(\mathrm{P}_{3}\right)$, and tuning transformer as shown in figure 3 . With the power meter, for which $\mathbf{M}_{g m}$ is required, connected to port 2 , tuner $\mathrm{T}_{y}$ is adjusted such that $\mathrm{P}_{3}$ vanishes. ${ }^{2}$ This power meter is next replaced by a moving short. In response to motion of the short, $\mathrm{P}_{3}$ will undergo cyclic variations of which the maximum, $\mathrm{P}_{3 M}$, and minimum, $\mathrm{P}_{3 m}$, values are observed.

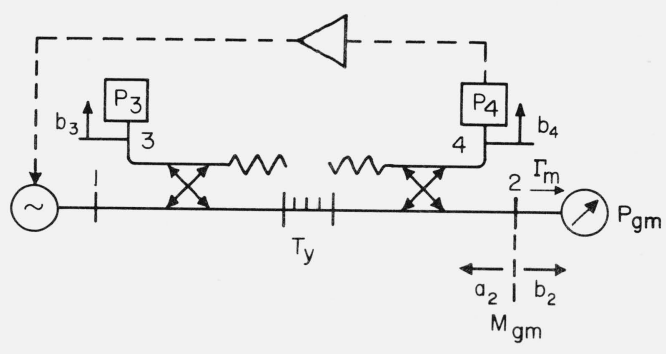

Figure 3. Addition of a second coupler and tuning transformer $\left(T_{y}\right)$ to permit the measurement of $M_{g m}$.

In the following section, it will be shown that $\mathrm{M}_{g m}$ is given by : ${ }^{3}$

$$
M_{g m}=1-\left[\frac{\sqrt{P_{3 M}}-\sqrt{P_{3 m}}}{\sqrt{P_{3 M}}+\sqrt{P_{3 m}}}\right]^{2}
$$

2 Ordinarily, the ratio of the power meter reading to $\mathrm{P}_{4}$ will also be obtained at this time as part of the overall calibration procedure.

3 In the event that $P_{4}$ is not constant $P_{3 M}$ and $P_{3 m}$ should be replaced by $\left(\frac{P_{3}}{P_{4}}\right)_{M}$ and $\left(\frac{P_{3}}{P_{4}}\right)_{m}$. 
It is to be emphasized that this procedure completely accounts for arbitrary power meter impedance, imperfect coupler directivity and impedance, and for connector imperfections, provided only that the connector dissipation is repeatable. ${ }^{4}$

It is of interest to note that the expression in the parenthesis is also that used to convert VSWR to reflection coefficient. It is thus possible to visualize the procedure in the context of a matched (reflectionless) generator, slotted line, and mismatched load. The slotted line is used to measure the load VSWR, from which the reflection coefficient, $\Gamma$, is obtained. The mismatch factor, $M$, is then computed from the equation:

$$
M=1-|\Gamma|^{2} \text {. }
$$

\section{Analysis}

It is the purpose of this section to provide the theoretical basis for the described procedure.

Beginning with the directional coupler of figure 2, a solution of the scattering equations yields:

$$
b_{2}=\frac{b_{4}}{D}-\frac{C}{D} a_{2}
$$

where $C, D$ are functions of the coupler scattering parameters, and the reflection coefficient of the power detector terminating arm 4, but not of the generator which feeds arm 1.

In general the equation for a source is written

$$
b_{2}=b_{g}+a_{2} \Gamma_{g}
$$

where $b_{g}$ is the "generator wave," and $\Gamma_{g}$ the source reflection coefficient $[8]$. Comparison of (4) and (5) indicates that: ${ }^{5}$

$$
\begin{array}{r}
\frac{b_{4}}{D}=b_{g} \\
-\frac{C}{D}=\Gamma_{\mathrm{g}} .
\end{array}
$$

Equation (4) may be solved for $b_{4}$ to obtain

$$
b_{4}=C a_{2}+D b_{2}
$$

Referring next to figure $3, b_{3}$ is also expressible as a linear combination of $a_{2}, b_{2}$

$$
b_{3}=A a_{2}+B b_{2}
$$

and taking the ratio of (9) and (8) leads to

$$
\frac{b_{3}}{b_{4}}=\frac{A \Gamma_{l}+B}{C \Gamma_{l}+D}
$$

where $\Gamma_{l}$ is the reflection coefficient of the termination on port 2 , i.e.,

$$
\Gamma_{l}=\frac{a_{2}}{b_{2}} .
$$

4 Impedance properties of the connector are not a consideration. For a more complete discussion see [1] and [7].

5 The (implicit) definitions for C, D have been chosen in such a way as to permit a ready comparison with a number of earlier results.

${ }^{6} \mathrm{It}$ is important to note that this adjustment of the ratio $B / A$ does not affect the values of $C, D$ since these are parameters only of the coupler on the right.

7 The short is assumed to be lossless. A theory which accounts for this loss has been developed; however the error due to this source, in this application, is negligible.
Equation (10) is the usual starting point in reflectometer theory [9].

By hypothesis, $T y$ is adjusted ${ }^{6}$ such that $b_{3}\left(P_{3}\right)$ vanishes when $\Gamma_{l}$ equals the power meter reflection coefficient, $\Gamma_{m}$. Therefore,

$$
B=-A \Gamma_{m}
$$

With the sliding short connected to the output port (2), $\Gamma_{l}=e^{i \theta}$, where $\theta$ is determined by the short position. ${ }^{7}$ Substitution in (10) yields:

$$
\begin{aligned}
\frac{b_{3}}{b_{4}} & =\frac{A e^{i \theta}+B}{C e^{i \theta}+D} \equiv \frac{B D^{*}-A C^{*}}{|D|^{2}-|C|^{2}}+ \\
& +\frac{(A D-B C) e^{i \theta}\left(D^{*}+C^{*} e^{-i \theta}\right)}{\left(|D|^{2}-|C|^{2}\right)\left(D+C e^{i \theta}\right)} .
\end{aligned}
$$

By inspection

$$
\left|\frac{b_{3}}{b_{4}}\right|_{\text {max }}=\left|\frac{B D^{*}-A C^{*}}{|D|^{2}-|C|^{2}}\right|+\left|\frac{A D-B C}{|D|^{2}-|C|^{2}}\right|
$$

and combining this with (7) and (12) leads to:

$$
\left|\frac{b_{3}}{b_{4}}\right|_{\text {max }}=\left|\frac{A}{D}\right| \frac{\left(\left|1-\Gamma_{m} \Gamma_{\theta}\right|+\left|\Gamma_{m}-\Gamma_{\theta}^{*}\right|\right)}{\left(1-\left|\Gamma_{\theta}\right|^{2}\right)}
$$

It is easy to show that the first term in the numerator exceeds the second if $\left|\Gamma_{g}\right|<1,\left|\Gamma_{m}\right|<1$; therefore:

$$
\left|\frac{b_{3}}{b_{4}}\right|_{\text {min }}=\left|\frac{A}{D}\right| \frac{\left(\left|1-\Gamma_{m} \Gamma_{g}\right|-\left|\Gamma_{m}-\Gamma_{g}^{*}\right|\right)}{\left(1-\left|\Gamma_{g}\right|^{2}\right)}
$$

Finally:

$$
1-\left[\frac{\left|\frac{b_{3}}{b_{4}}\right|_{\max }-\left|\frac{b_{3}}{b_{4}}\right|_{\min }}{\left|\frac{b_{3}}{b_{4}}\right|_{\max }+\left|\frac{b_{3}}{b_{4}}\right|_{\min }}\right]^{2}=1-\left|\frac{\Gamma_{m}-\Gamma_{g}^{*}}{1-\Gamma_{m} \Gamma_{g}}\right|^{2}
$$

and

$$
1-\left|\frac{\Gamma_{m}-\Gamma_{g}^{*}}{1-\Gamma_{m} \Gamma_{g}}\right|^{2} \equiv \frac{\left(1-\left|\Gamma_{g}\right|^{2}\right)\left(1-\left|\Gamma_{m}\right|^{2}\right)}{1-\left.\Gamma_{m} \Gamma_{g}\right|^{2}}
$$

This last expression will be recognized as the conversion factor between the available power and the net power delivered to the load $\Gamma_{m}[10]$. It is thus equal to $M_{g m}$.

\section{An Alternative Procedure for Measuring $M_{g m}$}

For the sake of completeness, the existence of an alternative procedure for measuring $M_{g m}$ should be noted.

Returning to figure 3,Ty is adjusted such that the ratio $P_{3} / P_{4}$ is constant with respect to the motion of a short at arm 2. Then it has been shown $[1,11]$ that $M_{g m}$ is given by:

$$
M_{g m}=1-\frac{\left[\frac{P_{3}}{P_{4}}\right]_{m}}{\left[\frac{P_{3}}{P_{4}}\right]_{s}}
$$

where the subscripts $m, s$ represent the values of $P_{3} / P_{4}$ with the meter and short connected respectively. 
As compared with the previous method, the prescribed adjustment of $T y$ is more difficult to realize, but once obtained is the same for all terminations (power meters). A more complete description is found in the references (e.g., $[1,7,9,11])$; the discussion which follows pertains entirely to the first procedure. Finally, it is possible to eliminate the tuning operation entirely if the phase difference between $b_{3}$ and $b_{4}$ is measured. This, however, is the subject of a paper to follow.

\section{Experimental Evaluation}

A series of tests have been made of this technique which will now be described. The initial objective was to demonstrate the performance potential in a simulated calibration hierarchy using "ordinary" waveguide hardware. To this end a frequency of $9 \mathrm{GHz}$ and the type " $\mathrm{N}$ " connector were chosen.

Referring to figure 1, six power meters and six "signal generators" were prepared. The calibration was propagated through the simulated hierarchy as described, and the last (sixth) item then compared directly against the first. This provided an indication of the error accumulation in the process. (It should be noted that an "absolute" calibration of the first item (standard) was not required.)

The components comprising the "signal generators" (figure 3) were in waveguide with a transition to type "N" at the output port. Although a recently improved version of the type " $\mathrm{N}$ " was incorporated in several of the components, a substantial number of the items had been in use for many years. The "equivalent generator" VSWR included values as large as 2.5. Finally, the powers $P_{3}, P_{4}$ were measured with Type II NBS bridges [6].

The "six level" experiment outlined above was repeated four times and gave error accumulations of 0.33 percent, -0.23 percent, 0.66 percent, and 0.43 percent. The mean of these values is 0.30 percent, while the standard deviation is 0.48 percent.

The design was also such as to permit a comparison of the relative error contributions of the individual components. Within the sensitivity of experiment, there was no deterioration in accuracy with the "older" connectors as compared with the more recent versions, or with the poorly matched generators as compared with those of small VSWR.

Although the above analysis has some intuitive appeal, considerably more information can be obtained through a more sophisticated analysis based on the method of least squares. Here an estimate of the standard deviation of a single calibration transfer from one meter, through a generator, to the next meter is obtained. For the first experiment this standard deviation, of a single calibration transfer, is 0.12 percent.

It was next decided to repeat the experiment with a group of "improved" Type N connectors (all of which were of the same model). The repeated experiment gave a standard deviation, for a single transfer, of 0.46 percent : four times the previous result. Examination of the results showed, however, that one of the connectors was contributing a major share of the total error. Fortunately, the experiment was designed in such a way that the effect of individual connectors could be both recognized and deleted. When this was done, the standard deviation became 0.22 percent.

The experiment was then repeated a third time with a different set of "improved" Type N connectors. The results were essentially identical to those obtained in the second run: one connector was defective, and when this was eliminated the standard deviation was 0.24 percent.

In the fourth experiment, the GPC-7 connector was substituted for the Type $\mathrm{N}$; this gave a standard deviation of 0.13 percent. Finally, the experiment was repeated a fifth time in waveguide using conventional flange connectors. This gave a standard deviation of 0.054 percent. In the fourth and fifth experiments, three "outlying observations" were discarded, but these were single measurements rather than all measurements associated with one connector as had been eliminated in experiments two and three.

Thus far the discussion has been primarily concerned with the random error; however, the quoted results of the first experiment raise the question of a possible systematic component as well.

Because the same measurement procedures are employed with both "standard" and "unknown," there is no apparent source of systematic error other than possible instabilities of the several components (bolometer elements, etc.) with respect to time. A further analysis of the results shows that the postulated absence of systematic error is not rejected with 95 percent confidence. Stated in other words, the "apparent" systematic errors observed in the experiments are easily explained by the random errors observed.

The results of the five experiments are summarized in table 1. Again it is emphasized that this method is, in theory, independent of impedance discontinuities in the connector. Thus these figures reflect only the nonrepeatability of the power dissipation at the connector interface.

Although the primary emphasis in the experiment was on dissipation repeatability, it is possible to infer something about the impedance repeatability from the repeatability of the mismatch factor $\left(M_{g m}\right)$.

Unfortunately, the design of the experiment was such as to provide only a minimal amount of information on

TABLE 1. Measurement results

(Ability to effect a single calibration transfer)

\begin{tabular}{|c|c|c|c|}
\hline Connector & $\begin{array}{l}\text { Estimate of } \\
\text { standard } \\
\text { deviation }\end{array}$ & \multicolumn{2}{|c|}{$\begin{array}{l}95 \% \text { Confidence } \\
\text { interval for } \\
\text { standard deviation }\end{array}$} \\
\hline $\begin{array}{l}\text { Type } \mathrm{N} \\
\text { (Mixed) }\end{array}$ & $0.12 \%$ & $(0.091 \%$ & $0.18 \%)$ \\
\hline $\begin{array}{l}\text { Improved Type } \mathrm{N} \\
\text { (Model 1) }\end{array}$ & $.22 \%$ & $(.16 \%$ & $.34 \%)$ \\
\hline $\begin{array}{l}\text { Improved Type } \mathrm{N} \\
\text { (Model 2) }\end{array}$ & $.24 \%$ & $(.18 \%$ & $.37 \%)$ \\
\hline GPC-7 $\ldots \ldots$ & $.13 \%$ & $(.10 \%$ & $.18 \%)$ \\
\hline Waveguide _.......... & $.054 \%$ & $(.042 \%$ & $.076 \%)$ \\
\hline
\end{tabular}


this point, but the results were consistent with expectation. In particular, the waveguide joint showed a marked improvement over the GPC-7, and the GPC-7, some improvement over the Type N. Perhaps of greater interest is the result that three of the Type $\mathrm{N}$ connectors showed a substantially poorer mismatch factor repeatability than was typical of the group; in only one case, however, was this accompanied by poorer dissipation repeatability.

Although it is not within the scope of this paper to describe these experiments in greater detail, it is anticipated that this will be included in a later paper. In brief, the pertinent conclusions are:

(1) For a single calibration transfer at NBS, standard deviations of 0.12 percent to 0.25 percent were observed in coax, and 0.054 percent in waveguide. Errors much larger than $\pm 3 \sigma$ were occasionally recorded. It appears that the type of connector is a significant factor, but this awaits confirmation. In retrospect it would have been desirable to have used a random ordering instead of segregating the several connector types into separate sequences. The initial objective, however, was to demonstrate the performance potential of the method, rather than make a comparative evaluation among different connector types.

(2) There is no evidence that the taking of several power readings and averaging the results contributed anything to the precision (other than as a guard against errors in data taking). Presumably, however, the precision would be improved by repeating the experiment "from scratch."

(3) No evidence of systematic error was observed during this experiment, which was of such size and precision that a systematic error of the order of 0.1 percent should have been detected. (Note, however, this paper deals only with the calibration transfer process, and says nothing about systematic errors in the reference standard.)

(4) Several of the connectors suffered from apparent problems in the area of dissipation repeatability. The source of this difficulty could not be recognized by a simple visual inspection.

\section{Summary}

A simplified method of disseminating power calibration has been described. This procedure may be regarded as a detailed example of the power equation concept. The key feature of this technique is the "direct" measurement of the mismatch factor $(\mathrm{Mgm})$ which eliminates the intermediate step of measuring the complex reflection coefficients. The performance potential was demonstrated in a series of experiments at $9 \mathrm{GHz}$.

Although the hardware requirements present no fundamental difficulty, the technique does call for certain equipment which is not the most common. In particular it is necessary to postulate either a stabilized (leveled) source (with respect to arm 4) or alternatively a direct method of obtaining $P_{3} / P_{4}$ is desirable. In addition, coaxial sliding shorts have found but limited application in the existing art. Whereas the method provides a substantial improvement in the accuracy potential with "conventional" hardware components, the associated bolometer bridges, etc. must be of high quality if this potential is to be achieved.

The measurement procedure is simple enough to envi- sion its use at the field level; here the component tolerances are usually the poorest, and the greatest projected benefits may be obtained. A series of measurements at NBS has demonstrated that it is now "possible" to propagate a power calibration through a typical calibration hierarchy with an error accumulation of 0.5 percent or less. (Obviously, however, unless this or a comparable technique is used, and appropriate quality control is maintained at each level, this potential will not be realized.)

Finally, the results of the experimental evaluation raises some rather serious questions relative to current trends in connector development. In particular, in terms of the requirements of this method and these experiments, several "improved" Type N connectors did rather poorly as compared with the "ordinary" Type N, while the GPC-7 was only on a par with it.

The reasons for the "poor" performance of the "improved" Type $\mathrm{N}$ is uncertain at this time. It may be associated with the use of stainless steel in its construction. In any case a further study of this subject is desirable.

The author expresses his appreciation to M. P. Weidman for the experimental results described above, to B. L. Joiner and P. V. Tryon of the Statistical Engineering Laboratory for their contribution to the design of the experiments and interpretation of results, and to L. E. Huntley for his contribution in the preparation of this manuscript.

\section{References}

[1] Engen, G. F., Power equations: A new concept in the description and evaluation of microwave systems, IEEE Trans. on Instrumentation and Measurement IM-20, No. 1, (Feb. 1971).

[2] Crawford, M. L., A new RF-DC substitution calorimeter with automatically controlled reference power, IEEE Trans. on Instrumentation and Measurement IM-17, No. 4, 378-384 (Dec. 1968).

[3] Engen, G. F., A refined X-band microwave microcalorimeter, J. Res. Nat. Bur. Stand. (U.S.), 63C (Eng. and Instr.), No. 1, 77-82 (July-Sept. 1959).

[4] Engen, G. F., A bolometer mount efficiency measurement technique, J. Res. Nat. Bur. Stand. (U.S.), 65C (Eng. and Instr.), No. 2, 113-124 (April-June 1961).

[5] Engen, G. F., Amplitude stabilization of a microwave signal source, IRE Trans. Microwave Theory and Techniques, MTT-6, No. 2, 202-206, (April 1958).

[6] Larsen, N. T., and Clague, F. R., The NBS type II power measurement system, Proceedings 25th Annual ISA Conference.

[7] Engen, G. F., An introduction to the description and evaluation of microwave systems using terminal invariant parameters, Nat. Bur. Stand. (U.S.), Monogr. 112, 25 pages (Oct. 1969).

[8] Kerns, D. M., and Beatty, R. W., Basic Theory of Waveguide Junctions and Introductory Microwave Network Analysis, (Pergamon Press, New York, 1967) p. 51.

[9] Engen, G. F., and Beatty, R. W., Microwave reflectometer techniques. IDS Trans. Microwave Theory and Techniques MTT-7, 351-355 (July 1959).

[10] Kerns, D. M., and Beatty, R. W., loc. cit., p. 56.

[11] Engen, G. F., A transfer instrument for the intercomparison of microwave power meters, IRE Trans. Instrumentation I-9, No. 2, 202-208 (Sept. 1960).

(Paper 75C2-317) 